\title{
Time-Lapse Imaging of Neural Stem Cells Exposed to Microgravity on the International Space Station
}

\section{Scott Olenych}

ZEISS Research Microscopy Solutions

scott.olenych@zeiss.com

\begin{abstract}
Astronauts who spend significant time in space may experience intracranial hypertension (pressure inside the skull), which often does not normalize upon return to Earth. This type of intracranial pressure can cause vision problems, headaches, glaucoma, and other serious health problems, making it a significant obstacle to long-duration space exploration missions. NASA is hoping that a better understanding of how central nervous system stem cells divide in microgravity will help lead to ways to protect astronauts from problems with intracranial pressure and to design adequate preventive measures. These studies examine cell growth and division of neural stem cells that experienced microgravity on the International Space Station and were returned to normal gravity prior to live-cell imaging studies to determine cell changes.
\end{abstract}

Keywords: neural stem cells, oligodendrocytes, microgravity, timelapse imaging, International Space Station

\section{Introduction: Unique Study Investigates \\ Multiplication of Human Nervous System Cells in Microgravity}

Dr. Araceli Espinosa-Jeffrey, a research neurobiologist at the Semel Institute for Neuroscience and Human Behavior at UCLA, has had a long-standing interest in making more neural cells faster. Her initial interest was for their use in transplantation research, and she has conducted experiments that observed a faster rate of cell growth in simulated microgravity $(\operatorname{sim}-\mu \mathrm{G})$ compared to that seen under standard Earth gravity conditions [1]. Believing that studies using microgravity will increase our understanding of the brain in health and disease, and in particular, to the discovery of new molecules and mechanisms impossible to unveil while in normal gravity conditions (1G), she sought and obtained funding from the National Aeronautics and Space Administration (NASA) to place an experiment using human brain cells aboard the International Space Station (ISS).

For the NASA studies, human brain cells were flown to the ISS on the SpaceX CRS-16 mission with the goal of gaining a better understanding of how neural stem cells grow and develop in true microgravity (Figure 1). Understanding cell response to true microgravity conditions is key to discovering more about the serious issues of intracranial hypertension affecting astronauts returning from space. In the future, information gathered may also be used to further cell replacement therapies for people with neurological disorders or neurological diseases. To study the effects of microgravity on the cells that had been on the ISS, Dr. Espinosa-Jeffrey and her research team conducted time-sensitive 72-hour timelapse imaging to examine proliferation, migration, and other features of cells as they readapted to terrestrial gravity. The goal was to confirm and expand the results from the sim- $\mu \mathrm{G}$ microgravity experiments they had conducted and to learn more about cells in space microgravity, including the effects on different cell types [1,2].

NASA's particular interest in funding the research was spurred by its desire to learn more about the role increased division of these cells in space may play in the intracranial hypertension (pressure inside the skull) observed during human spaceflight, which often does not normalize when astronauts return to Earth. This type of intracranial pressure can cause vision problems, headaches, glaucoma, and other serious health problems, making it a significant obstacle to long-duration space exploration missions. NASA is hoping that a better understanding of how central nervous system stem cells divide in microgravity will lead to the design of adequate preventive measures and protection for astronauts from problems with intracranial pressure.

The work was similar to a study investigating oligodendrocyte progenitors (OLPs) grown in simulated microgravity and designed to investigate the effects of space microgravity on the global secretome of these cells including molecules important for cell-cell communication, function, stemness, and differentiation. The knowledge gained from these experiments will contribute to a better understanding of how stem cell growth is affected by gravity at the molecular level and may help advance neural stem cell technologies that would be useful in wound healing, tissue regrowth, and organ culture.

When OLPs used in this study were cultured in sim- $\mu \mathrm{G}$ on the ground, they divided faster than cells cultured under normal gravity conditions [1]. Espinosa-Jeffrey has reported that neural stem cells also proliferated more in sim- $\mu \mathrm{G}$ [3] and space microgravity [4]. Studying the reasons why, and the mechanisms causing these cells to divide faster in microgravity, could pave the way to breakthroughs in stem cell production for cell replacement therapies. Finding ways to generate more stem cells is urgent because there is currently no way to quickly produce the large numbers of "self" stem cells needed for the many cell replacement therapies that are showing such promise for people with neurological disorders, neurological diseases like multiple sclerosis, and developmental disabilities.

In addition, using one's own cells without grafting donor cells and tissues opens the possibility that a neural stem cell donor could be his or her own recipient, eliminating risks of rejection. Such an approach could be used for repair of head and spinal injuries, as well as other cell replacement therapies. In addition to her work with NASA, Dr. Espinosa-Jeffrey would like to work on these issues with the Center for the Advancement of Science in Space, Inc. (CASIS, also known as the ISS 


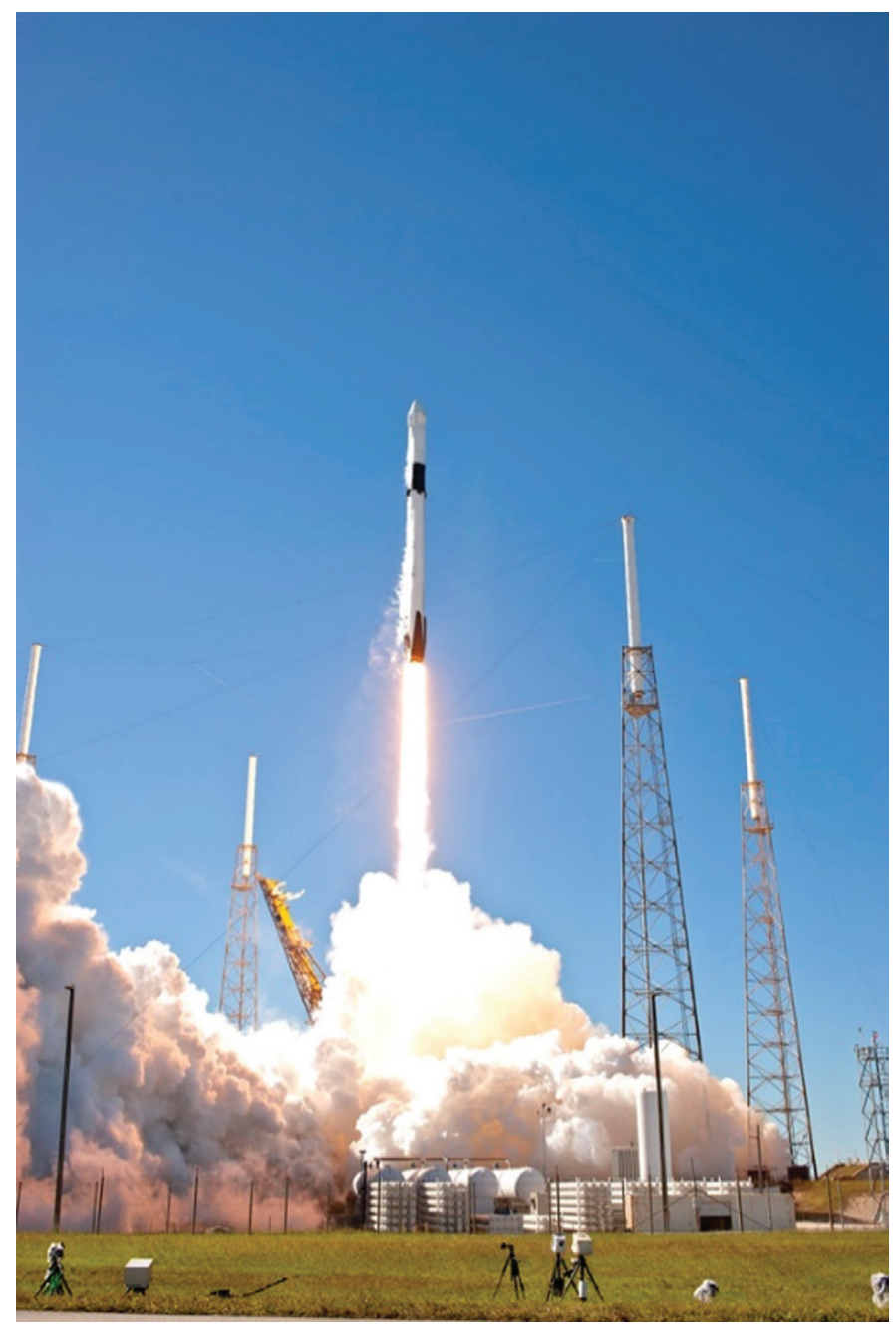

Figure 1: SpaceX CR-16 lift-off with human neural stem cell experiments aboard. The experiments to test whether cell proliferation is increased in microgravity were designed and implemented by Dr. Espinosa-Jeffrey and managed by NASA's Ames Research Center, Moffett Field, CA.

National Laboratory), which is pursuing research on the effects of microgravity on cells to better study diseases and therapies aimed at prevention and treatment.

\section{Methods and Materials}

The NASA experiment, dubbed BioScience-04: The Impact of Real Microgravity on the Proliferation of Human Neural Stem Cells and Derived-Oligodendrocytes, was launched in the SpaceX CRS-16 Dragon capsule in December 2018. Two types of cells, neural stem cells and oligodendrocyte progenitor cells (Figure 2), were studied in space. Neural stem cells produce all three major nervous system cell types: neurons, and the two kinds of cells that support neurons, astrocytes and oligodendrocytes. Neural stem cells also produce progenitor cells, which are like stem cells because they divide to produce new cells but are more limited because they cannot divide and produce new cells indefinitely. One kind of progenitor cell produced by neural stem cells is the OLP cells, which become mature oligodendrocytes (Figure 3).

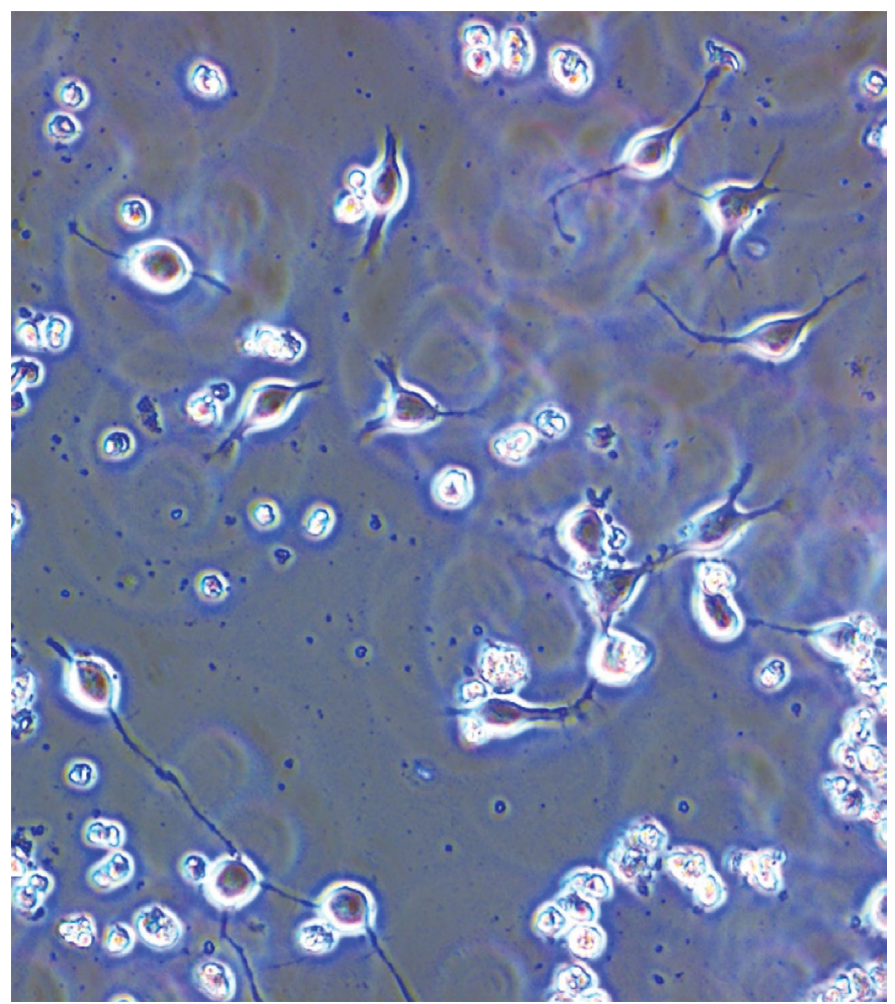

Figure 2: Micrograph of human neural stem cells cultured for 45 days in normal gravity [7]. Credit: UCLA, Dr. Espinosa-Jeffrey.

Oligodendrocytes produce electrical insulating membranes known as myelin sheaths that coil around neuronal axons allowing signals to move along nerves at normal speeds, making these cells vitally important to nervous system health (Figure 4). Determining if these important cells divide into two daughter cells faster in the microgravity environment of space will help scientists study the cell-signaling pathways that determine cell function, proliferation, and differentiation.

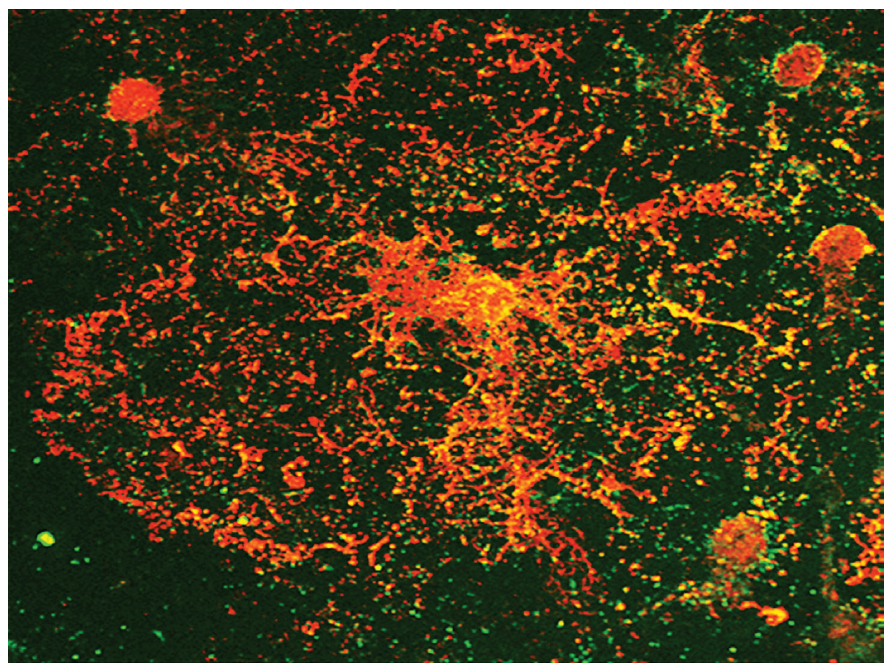

Figure 3: Mature oligodendrocyte in maturation medium expressing myelin basic protein (red) and sulfatides (green). Credit: UCLA, Dr. Espinosa-Jeffrey. 


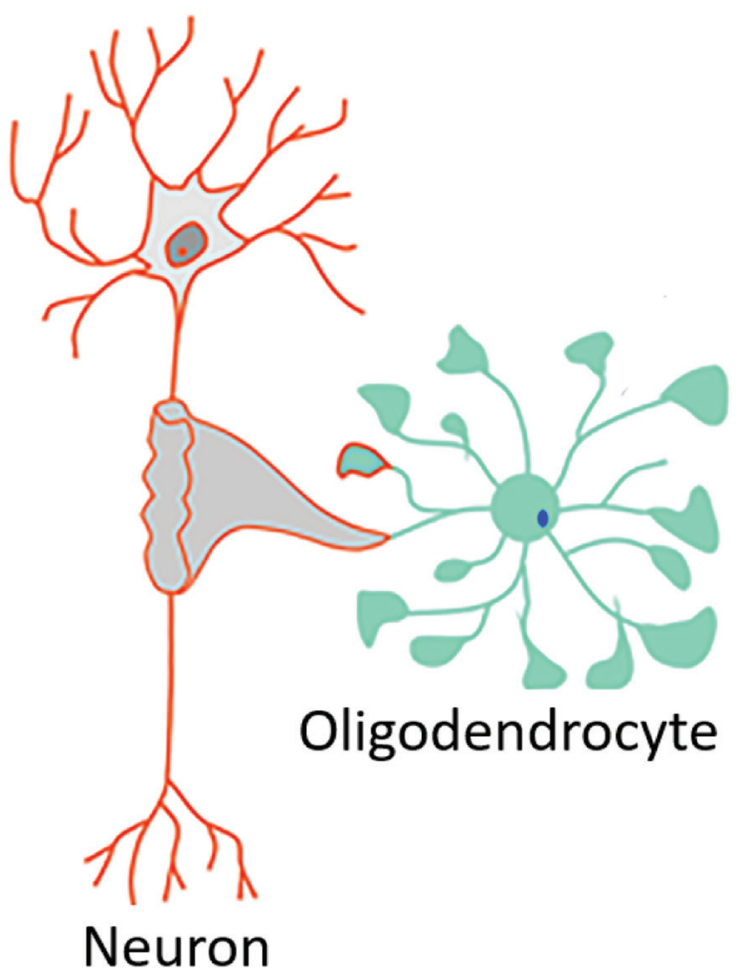

Figure 4: Diagram of an oligodendrocyte wrapping around a neuron's axon.

The two cell types sent to the International Space Station were maintained in cell culture hardware developed by Airbus-Kiwi (Friedrichshafen/Germany, formerly .kiwimicrogravity, currently Yuri, https://www.yurigravity.com/ our-service) (Figure 5) and then installed in the Space Technology and Advanced Research Systems (STaARS)-1 Experiment Facility, where the cells were given time to grow and divide. Dr. Espinosa-Jeffrey designed the experiments and inspired the STaARS team to design the self-contained cell culture box. Automated control of the experiments, including culture media exchanges, was conducted from Houston, TX so astronauts would not have to invest time on this task.
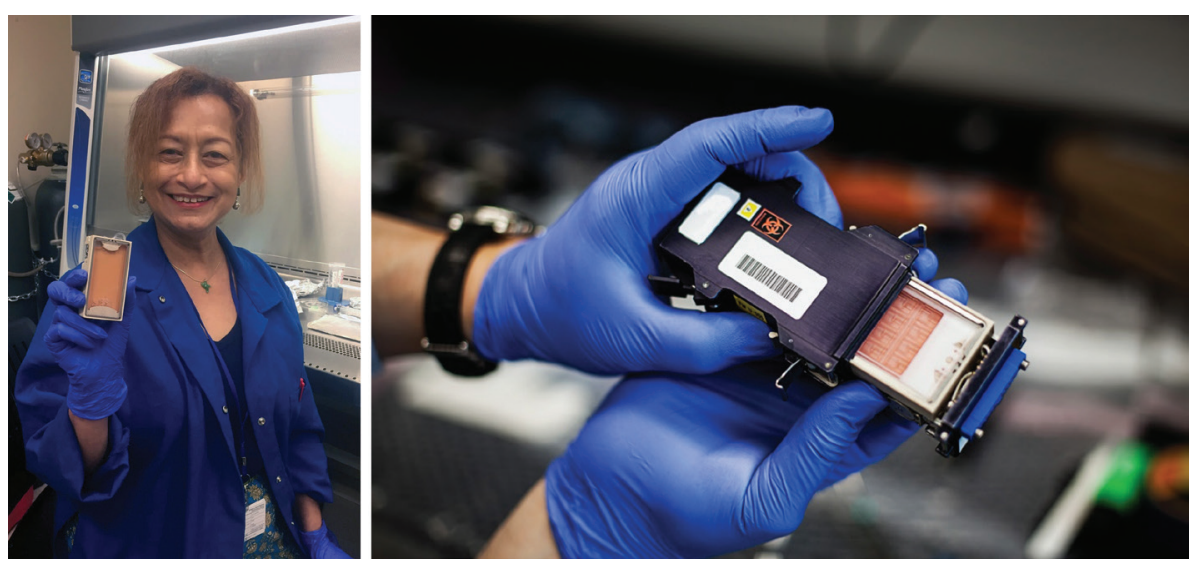

Figure 5: (Left) Dr. Espinosa-Jeffrey holding the Airbus-Kiwi (Yuri) cell culture automated chamber [7]. (Right) Airbus-Kiwi (Yuri) automated cell culture chamber.
Due to delays, cells were maintained on the ISS for 39.3 days instead of the 35 days originally anticipated. After splashdown, transport to Long Beach airport, and delivery to UCLA, the human neural stem cells were retrieved from the hardware, plated onto poly-d-lysine-coated flasks in a stem cell chemically defined medium [5], and allowed to recover from space flight. Researchers then began measuring how much the cells had divided while in space by using the Automated Type IV units from Yuri.

After space flight, cells were seeded onto microscopy compatible flasks and $72 \mathrm{~h}$ continuous time-lapse microscopy allowed analysis of cell proliferation and measurement of the cell cycle duration [6]. Moreover, using the same approach, Dr. Espinosa-Jeffrey's team is currently determining the effects of the secretome of stem cells flown onto space on $1 \mathrm{G}$ (naïve) cells and identifying the proteins secreted.

\section{Dedicated Microscope Resources Needed for Crucial Time-Lapse Imaging}

Time-lapse imaging and cell division and proliferation studies were conducted with the ZEISS Axio Observer 7 fully motorized inverted research microscope equipped with Definite Focus 2, ZEISS Axiocam 506 monochrome camera with ZEISS ZEN software, and ZEISS Full Incubation XL chamber for temperature and $\mathrm{CO}_{2}$ control with a motorized scanning stage (Figures 6 and 7). Live-cell imaging equipment was essential as all data had to be collected within 72 hours using a controlled temperature and gas environment equivalent to what cells would experience in a standard tissue culture incubator. The cells had to be kept at the temperature of the human body with in vivo conditions to mimic their native environment to avoid the risk of sample degradation over time and a potentially diminished ability to detect subtle effects of the spaceflight environment. Completion of the analysis was especially time critical. For extended time-lapse studies, images were collected every 10-15 minutes at each of 6 to $8 x y z$ positions for 72 hours (Figure 7). From the position list several time-lapse movies were created demonstrating cell growth and proliferation, as well as other features such as migration.

\section{Discussion}

It was extremely important to begin analysis immediately, so there would be no chance of missing any changes to the cells. It was important to show that cells behaved as they did in simulated microgravity, where some cells move slightly faster. Analysis of data from time-lapse images showed that these cells had proliferated post-flight at comparable rates to control cells growing in normal gravity. For further studies, Dr. Espinosa-Jeffrey's team is now characterizing the secretome of space flight cells for novel features that they have not observed in ground control cells or in their simulated microgravity studies.

The Espinosa-Jeffrey team, using timelapse microscopy, has recently reported 


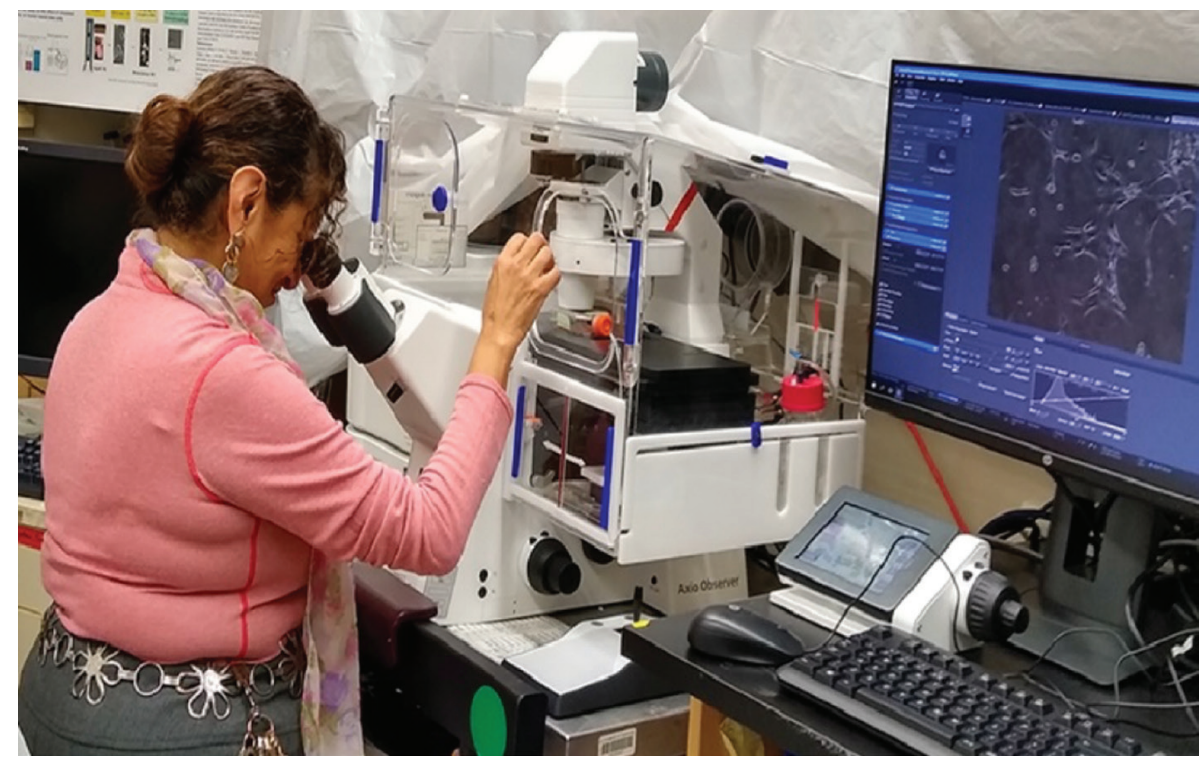

Figure 6: Dr. Espinosa-Jeffrey imaging with ZEISS Axio Observer 7.
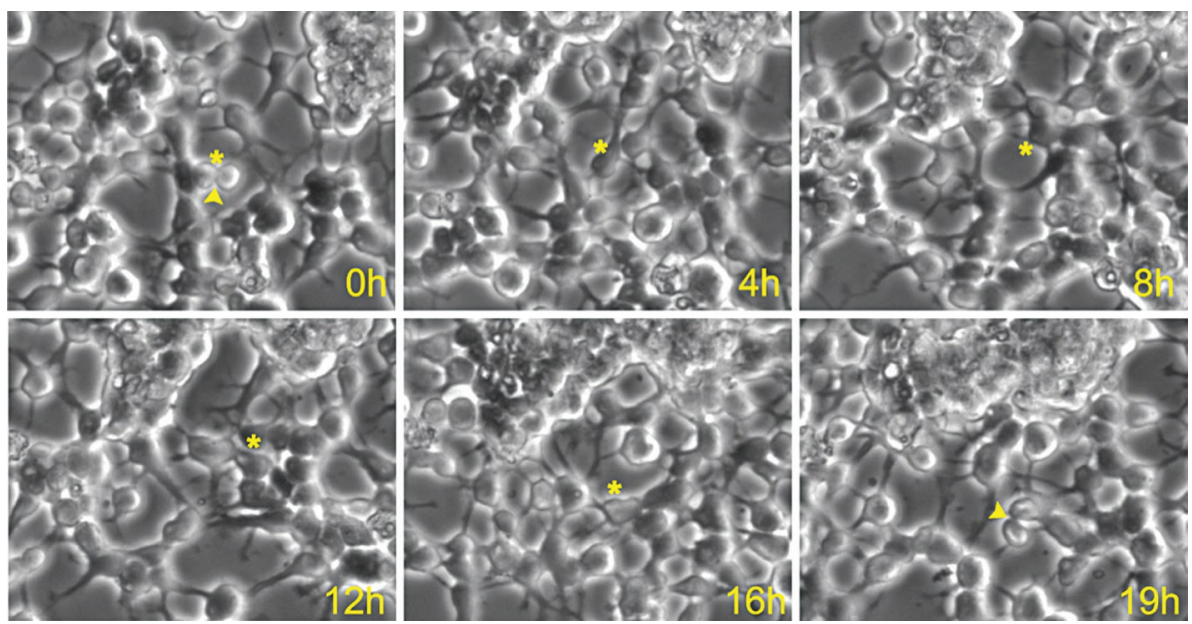

Figure 7: Proliferation of neural stem cells cultured post-exposure to zero gravity showing cell division (asterisk and arrowhead).

that neuronal stem cells that have flown in space become neurons when cultured in the appropriate conditions. They have also used the ZEISS LSM 800 confocal microscope to confirm their neuronal nature [6].

\section{Conclusion}

The information reported here is very much a work in progress, and the proliferation of neural stem cells in space must be studied in more detail to determine the possible relationship to the visual impairment and intracranial pressure syndrome exhibited by astronauts in space and upon their return to Earth. Learning more is essential to be able to design effective countermeasures for astronauts working onboard the ISS and for longterm space travel. The next phase of the work will be performed on SpaceX-21, a commercial resupply service mission to the ISS using the new Dragon 2 spacecraft, planned for October 2020.

\section{Acknowledgments}

We are grateful to the ZEISS team members who supported the live microscopy needs for the post-flight part of this project: Mark Mobilia - Account Manager for UCLA; Dr. Neeraj Gohad - Life Sciences PASS Manager, West; Dr. Ben Ng - Field Applications Scientist; Kevinne Aguilar - Local Service Engineer; Stefan Gross - Regional Sales Manager West, US; Tanya Mahboob - Demonstration Inventory Coordinator. Without their invaluable support, this part of our study would not have been possible. With the ZEISS timelapse system we were able to capture unique properties displayed by human neural cells after space flight. Thanks to Dr. Amy Gresser. Elizabeth Pane, and Medaya Torres for their help with the implementation of the study; The NASA Space Biology Project team at Ames Research Center including Dr. David Tomko, Dr. Kevin Sato, Elizabeth Taylor, ARC Space Biology Project Manager, and the support personnel at the Space Station Processing Facility at Kennedy Space Center; to Karin Perkins, Diana Ly, and Tino Hernandez for their invaluable support; to Maria Birlem and Chriss Bruderrek from Yuri, and STaARS team members Tom Kyler, Craig Walton, and BreAnne MacKenzie for supporting the flight implementation; to Uli Kuebler from Airbus Defense and Space, who introduced us to the flying hardware used for this study; and to the IDDRC Cell Culture Core.

\section{Funding}

We thank NASA Space Biology for Grant NNX15AB43G; the IDDRC Cell Culture Core is supported by NIH/ NICHD grant number U54HD087101-05.

\section{References}

[1] A Espinosa-Jeffrey et al., PLoS One 8 (2013) e76963 1-10.

[2] A Espinosa-Jeffrey et al., J Neurosci Res 94 (2016) 1434-50.

[3] J Green et al., "The Implications of Microgravity on Cell Morphology and Proliferation of Stem Cell Progenies Towards Astronaut Health," International Astronautical Federation (IAF), IAC-16-A1,7,9,x35726 (2016), Guadalajara, Mexico. http://iafastro.directory/iac/paper/id/35726/ summary.

[4] A Espinosa-Jeffrey et al., Applied Sciences (2020) (in press).

[5] A Espinosa-Jeffrey et al., J Neurosci Res 69 (2002) 810-25.

[6] C Cepeda, Appl Sci 9 (2019) 4042.

[7] NASA, About Bioscience-04 (SpaceX-16). https://www. nasa.gov/ames/research/space-biosciences/bioscience4-spacex-16. 